\title{
Mercury content of the human lens
}

\author{
J. D. ABRAMS AND U. MAJZOUB \\ Institute of Ophthalmology and Moorfields Eye Hospital, London
}

In patients receiving prolonged miotic therapy, using drops containing phenylmercuric nitrate as preservative, the anterior lens capsule develops an appearance which is identical to that of mercurialentis (Abrams, I963), a condition extremely common amongst those who work in an atmosphere of mercury. Only one analysis for mercury has been carried out hitherto on a lens showing mercurialentis (Atkinson and von Sallmann, 1946) and in this case the presence of mercury was reported without an exact figure for the amount detected.

Some doubt has, therefore, existed whether mercury is really present in the lens in such cases or whether the clinical appearance is due to some change in the capsule of the lens induced by mercury.

The fact that only one lens has been so analysed indicates the paucity of clinical material available and the technical difficulties in performing tests for very small quantities of mercury. Few lenses do in fact come to analysis because most patients with mercurialentis retain perfectly normal vision. Recently an opportunity has arisen to analyse the lens of a patient with the appearance of mercurialentis due to prolonged miotic therapy and the associated exposure to phenylmercuric nitrate; advantage has been taken of the method of activation analysis which is capable of detecting extremely small quantities of mercury.

\section{Method}

The method chosen was based on activation, by neutron irradiation, of the samples to induce the reaction $\mathrm{Hg}^{196}$ ( $\mathrm{n}, \gamma$ ) $\mathrm{Hg}^{197}$ followed by gamma-spectrometry to measure the $0 \cdot 08 \mathrm{MeV}$ gamma rays and comparison of their intensity with those from mercury standards of known weight given the same irradiation. In view of the low levels expected and the interference of high levels of sodium and chlorine activities usually found in irradiated biological samples, a simple chemical separation scheme was used rather than non-destructive analysis. This also gave the advantage of eliminating possible geometry errors when dealing with low energy gamma emitters.

The procedure used was to seal the samples after weighing them in silica ampoules, taking care to avoid overheating them and causing possible mercury losses. Standards were prepared using weighed amounts of mercuric nitrate solution made to $100 \mu \mathrm{g}$. $\mathrm{Hg} / \mathrm{ml}$, also sealed in silica. Samples and standards were irradiated in the BEPO reactor in a flux of $10^{12}$ neutrons $/ \mathrm{cm} .{ }^{2} / \mathrm{sec}$. for $24 \mathrm{hrs}$. After a short decay period the sample ampoules were cooled in liquid nitrogen, and then broken and rapidly transferred to a reflux distillation apparatus, where the samples were charred with sulphuric acid and wet-oxidized with nitric acid in the presence of $20 \mathrm{mg}$. inactive mercury carrier. Care was taken to prevent mercury losses at all stages, and after oxidation the solution was allowed to cool and then neutralized with sodium carbonate. The mercury was then precipitated by addition of $\mathrm{NH}_{4} \mathrm{~S}_{\mathrm{x}}$ and the precipitate spun off and washed. The precipitate was then dissolved in $\mathrm{H}_{2} \mathrm{~S}-$ saturated caustic soda and reprecipitated by boiling with $\mathrm{NH}_{4} \mathrm{NO}_{3}$ solution. Standards were treated in exactly the same way, and after washing the final precipitates were slurried onto weighed counting trays with alcohol, reweighed to determine the chemical yields, and then counted on a gamma spectrometer using a $3^{\prime \prime} \times 3^{\prime \prime}$ thallium-activated sodium iodide detector. Counting data 
were collected over a period of several days in order to verify that the gamma peaks obtainef decayed with a half-life of 65 hours and thus check radiochemical purity. Results were obtained bo comparing peak areas for samples and standards at an arbitrary point on the decay curves obtained

\section{Results}

The mercury content of three human lenses is reported in this paper. These lenses were a $\frac{\overline{\bar{p}}}{}$ cataractous. One showed the appearance of mercurialentis and came from a patient wh $\mathbb{8}$ had been using miotics containing phenylmercuric nitrate $0 \cdot 004$ per cent. for several yearof for glaucoma. The other two lenses did not have the appearance of mercurialentis, and came from patients with senile cataract only. The lens from the glaucoma patient showedi I .08 parts per million of mercury, and the other two lenses showed 0.08 and 0.03 parts pew million respectively.

The lenses were removed by identical techniques by one of us (U.M.) and care wais taken to use no local medications containing phenylmercuric nitrate as a preservative. Alpha-chymotrypsin was used and the lens was delivered "head-first" in order to avoieb any instrumentation to the central region of the anterior lens capsule.

\section{Comment}

The technique used for estimating mercury is probably accurate to within \pm 5 to ro peq cent. and the limits of determination of mercury by activation analysis are between $\mathrm{IO}^{-\delta}$ and $10^{-10} \mathrm{~g}$., a range which is very much below the quantities reported here. The mercury content of the suspected mercurialentis lens is therefore some twenty times higher than the content of the senile cataractous lens with most mercury in it.

Little is known about trace elements of the human lens, but in other parts of the bod 0.05 parts per million of wet weight is about the highest mercury content which has bee recorded. The figure of $\mathrm{I} \cdot 08$ parts per million must therefore be considered pathologicall high for the lens showing the appearance of mercurialentis. This is particularly so $\mathrm{i} \overrightarrow{\mathrm{g}}$ view of the fact that the mercury content relates to the lens as a whole and if, as now seem reasonable to conclude, the mercury is present in a small area of the anterior capsule, the concentration at that site must be considerably greater.

It is interesting to speculate on the precise cause of the beautiful appearance of mero curialentis on the slit lamp. Assuming that a human lens weighs about $200 \mathrm{mg}$., th amount of mercury present in the mercurialentis sample would be about $0 \cdot 2 \mu \mathrm{g}$. This exact thickness of a film produced by this amount of mercury in an area of perhaps 3 sci $\mathrm{mm}$. will depend upon the form in which the mercury is present. As it is known that mercury can be deposited from solutions of phenylmercuric nitrate, the film itself may bs composed of metallic mercury absorbed onto the capsule. Should this be so, the thickness. of the film works out somewhere in the region of $0 \cdot 01 \mu$, some fifty times smaller than the wave length of mid-spectral visible light. It is therefore hardly to be expected that this would present any significant obstruction to the transmission of light from aqueous humoum through the lens.

According to Heavens (1968), a world authority on the optical properties of thin film a layer of mercury of this thickness could be expected to have a slight granularity rathey than to appear as a true film, and according to the particle size coloured effects might be obtained from oblique illumination. These features are well known clinically in all the metalloses which affect the lens. It appears to be impossible to identify the chemic nature of the film merely by inspection of its optical properties, because these depend of the characteristics of the particles rather than on the chemical composition. 


\section{Summary and conclusions}

A cataractous lens was removed from a patient also suffering from chronic glaucoma. Miotic drops containing phenylmercuric nitrate 0.004 per cent. as a preservative had been used three times a day for 4 years and the lens showed the appearance of mercurialentis. 'It was found to have a significantly higher' mercury content than that of two other cataractous lenses. Although it has not been possible to identify the precise site in the lens where this mercury is localized, these analyses suggest that the appearance of mercurialentis is due to the deposition of either metallic mercury or a compound containing this metal.

The authors wish to express their thanks to Prof. Barrie Jones, from whose patients the material analysed in this paper was obtained, and to Dr. D. Gibbons of the United Kingdom Atomic Energy Authority who gave valuable technical advice, carried out the investigations of mercury, and is largely responsible for the account of the methods used for so doing which are included above.

\section{References}

ABRAMS, J. D. (1963) Trans. ophthal. Soc. U.K., 83, 263

atkinson, w. s., and sallman, L. von (1946) Trans. Amer. ophthal. Soc., 44, 65

heavens, o. (1968) Personal communication 